\title{
IL DIO DELL'ARISTOTELE PERDUTO
}

\author{
Barbara Botter \\ PUC-RJ
}

\begin{abstract}
Aristotle's attitude with respect to opinions about the gods is disconcerting in several ways. In contrast with the preserved works, the fragments of the lost dialogues, in particular On Prayer and the book III of De Philosophia, seem to reflect an intention to conceive the relation of the humanity to the divinity, with Metaphysics ignores. The presence of a dialogue On Prayer is a proof of the idea that the gods should be honoured, a provision on which one insists only if one thinks that the surpassing nobility of the gods must be respected. This assertion collides head on with the theological interpretation often given to Metaphysics Lambda. The latter, it has been said, uses more scientific arguments and abandons the "popular" theological thinking. This was formerly reason to suspect that Aristotle did not compose a dialogue On Prayer. We think better to refer to the facts and not seek to displace Aristotle's piety. In this paper we will attempt to show that there is not reason to suspect of the authenticity of the lost dialogue On Prayer, to give an interpretation of the fragment and to show the relationship between the Metaphysics Lambda and the exoteric work.
\end{abstract}

Keywords: god, nous, theology, metaphysics.

Resumo: A postura de Aristóteles com relação aos deuses é, às vezes, desconcertante. Diferentemente das obras do Corpus aristotelicum, os Diálogos essotericos, em particular 0 Peri Euches, parece testemunhar a intenção por parte de Aristóteles de pensar uma relação estrita entre 0 homem e a divindade, idéia esta que a Metafísica ignora. A existência de um dialogo Peri Euches seria sinal da persuasão que os deuses devem ser honrados e tal cuidado deriva do juízo que a nobreza dos deuses deve ser respeitada. Esta asserção não concorda com a interpretação tradicional das passagens teológicas de Metafísica. É reconhecido que a Metafísica utiliza argumentações mais cientificas e abandona 0 pensamento irrefletido da divindade. Inicialmente esta foi uma das razões que levaram os interpretes a colocar em duvida a autenticidade do dialogo Peri Euches. Achamos melhor nos manter fieis aos textos e atribuir à Aristóteles a "piedade" que ele merece. 0 presente estudo tentará mostrar que não há razão de duvidar da autenticidade do escrito essoterico. 
Tentaremos dar uma interpretação do fragmento do dialogo e mostrar a relação entre este texto e as passagens teológicas de Metafísica Lambda.

Palavras-chave: deus, nous, teologia, metafísica.

Aristotele è senza dubbio il filosofo antico più utilizzato dai teologi cristiani e musulmani come base filosofica per la loro visione monoteistica della realtà. I cristiani dapprima privilegiarono Platone e il neoplatonismo, si veda il caso notissimo di Agostino, ma poi, al seguito dei musulmani, che avevano fatto di Aristotele una specie di secondo Maometto, ne fecero anch'essi "il Filosofo" per eccellenza, per esempio Tommaso d'Aquino, e "il maestro di color che sanno", secondo l'espressione di Dante. È noto che, secondo una tradizione araba, Aristotele sarebbe apparso in sogno al califfo Al-Mamun e gli avrebbe detto: "Il tuo dovere è dichiarare l'unicità di Dio", cioè precisamente la verità fondamentale dell'islam. I musulmani videro infatti nel primo motore immobile, di cui Aristotele mostra la necessità nel libro XII della Metafisica, 1'unico Dio, creatore e signore del cielo e della terra, e quindi interpretarono questo libro come una specie di teologia, seguendo del resto in questo i commentatori greci tardo-antichi. A dire il vero essi dovettero trovare un po' scarse le indicazioni teologiche fornite da Aristotele nel libro in questione, perché pensarono di confezionare un'opera intitolata Teologia di Aristotele, desumendone il contenuto dalle opere ben più ricche, in questo senso, di Plotino. E i cristiani furono tratti in inganno da questo falso, confermandosi nell'idea che Aristotele fosse il creatore della teologia naturale o razionale. Dante, da sommo poeta qual era, riuscì a presentare il motore immobile, che "muove in quanto amato", come "l'Amor che muove il sole e 1'altre stelle".

Naturalmente la differenza tra il primo motore immobile di Aristotele e il Dio della Bibbia, concordemente adorato da cristiani e musulmani, era sotto gli occhi di tutti; ma essa divenne un pretesto per attaccare Aristotele, accusato di avere professato un concetto di Dio troppo astratto, meccanico, impersonale, egoista. In tale opera di denigrazione si scatenò una nobile gara fra cristiani e musulmani, che toccò i suoi vertici in campo musulmano col teologo Al-Gazali, nemico dei filosofi aristotelizzanti, e in campo cristiano con Lutero, noto per avere ricoperto il povero Aristotele degli insulti più feroci. Ancora oggi i musulmani e i cristiani integralisti non possono soffrire il "Motore immoto" di Aristotele, dichiarando che tale espressione fa pensare a un autocarro in panne e opponendogli, con Pascal, "il Dio di Abramo, di Isacco, di Giacobbe e di Gesù Cristo".

Sul tema del sentimento religioso nutrito dal filosofo di Stagira gli storici si mantengono su posizioni contrastanti. Cornelia de Vögel stima che la 
vita personale di Aristotele, così come il suo pensiero, siano estranei ad ogni sentimento religioso ${ }^{1}$. W. Bröcker arriva a giudicare l'aristotelismo una sorta di ateismo ${ }^{2}$. Secondo D. Babut "entre la théologie... et un ensemble de croyances mythiques.... il n'est point de place... pour une authentique religion, ...une veritable piété" 3 , e W. Wieland ritiene che nei primi anni della sua attività, Aristotele si sia abbandonato ad una sorta di "teologismo popolare", e abbia sacrificato le sue più radicate e genuine credenze alle differenti divinità mondane. La conclusione che si deve trarre è che le testimonianze che attribuiscono ad Aristotele un sentimento religioso non sono autentiche o, nel caso in cui lo fossero, devono essere attribuite all'Aristotele giovane, la cui interpretazione non contribuisce ad una migliore comprensione del Corpus pervenutoci.

Secondo L. Elders, al contrario, il Nostro avrebbe nutrito una profonda e fervente convinzione religiosa ${ }^{4}$, e W. Jaeger non nasconde la sua ammirazione per il grandioso tentativo promosso da Aristotele al fine di porre su basi sicure il problema di dio 5 . A. H. Chroust, qualche anno più tardi, notò che i trattati essoterici Sulla Filosofia e Sulla Preghiera difendevano un gran numero di proposizioni religiose o teologiche che $\mathrm{i}$ trattati del Corpus ignoravano, ma che supponevano conosciute e acquisite $^{6}$. Lo stesso giudizio risuona nelle parole di A. J. Festugière. Il carattere religioso delle opere essoteriche, secondo l'autore, si misura dal fatto che il filosofo greco cerca di render conto della credenza agli dei, di giustificare il rispetto "que l'on doit avoir a l'égard du cosmos et de montrer pourquoi les dieux célestes sont a adorer"7.

Ora, in relazione ai trattati essoterici, nonostante il successo che arrise loro al tempo di Aristotele e nel periodo che seguì immediatamente la morte del filosofo, essi scomparvero nei secoli successivi all'impresa editoriale di Andronico di Rodi. Ciononostante, noi siamo persuasi che se non si tiene conto delle dottrine contenute nelle opere pubblicate e se ci si basa esclusivamente sull'Aristotele del Corpus, si presenterà, dal punto di vista storico, un Aristoteles dimidiatus. Secondo Jaeger e la corrente evoluzionista

1 De Vögel, 1970, p. 17

2 Bröcker, 1964, p. 221

${ }^{3}$ Babut, 1974, p. 130.

${ }^{4}$ Elders, 1966, p. 95.

5 Jaeger, 1955, p. 162.

${ }^{6}$ Cfr. Chroust, 1977, pp. 129-133.

${ }^{7}$ Festugière, 1949, p. 229. 
Aristotele avrebbe pubblicato quelle opere da giovane, ossia finché era rimasto allievo di Platone, data la componente teologica in esse presente. La teologia platonica che le ha influenzate si sarebbe poi mutata in metafisica, una volta che Aristotele avesse maturato una linea di pensiero autonoma e originale. Questa tesi, parsa per molto tempo accettabile, oggi non regge più e le opere pubblicate non possono essere considerate appena delle opere giovanili. A prescindere dal fatto che per un lungo periodo queste opere siano quasi scomparse dalla circolazione, non è possibile oggi ignorarne l'esistenza. La conclusione che ci pare si debba trarre è la seguente: come non è possibile leggere i dialoghi platonici senza tener conto della tradizione indiretta che ci informa sulle "dottrine non scritte", analogamente non è più lecito, dal punto di vista storico ermeneutico, considerare il Corpus aristotelicum come autarchico e giudicare le opere essoteriche una appendice trascurabile di esso.

In relazione al sentimento religioso di Aristotele, noi partiamo dal presupposto che la teologia non si sia presentata ad Aristotele nei termini e nella forma in cui si presenta allo studioso moderno. In generale, siamo persuasi che Aristotele non affrontò l'indagine teologica con l'ausilio e gli strumenti della teologia razionale, ossia non fondò l'esistenza degli dei attraverso una teologia speculativa, e neppure all'interno di un contesto monoteista come quello della tradizione giudaico cristiana.

Il problema dell'esistenza degli dei non viene affrontato da Aristotele con gli stessi strumenti concettuali con cui sarebbe stato posto da un apologista cristiano. Il tentativo di Aristotele non è identico a quello della filosofia occidentale, quando essa si domanda, nel contesto monoteista della tradizione cristiana, se esiste un ente che possa realmente essere chiamato Dio. In un certo senso è una operazione diametralmente opposta alla preoccupazione fondamentale di questa tradizione, che risale a Sant'Agostino e ai primi apologisti cristiani. E questo per almeno due ragioni:

gli apologisti cristiani, per difendere "il Dio", erano abituati a considerare gli dei del paganesimo delle "false divinità";

la seconda ragione è la più importante: il motivo che spinse gli apologisti a creare delle prove sull'esistenza di Dio fu il timore di scoprire che la divinità non esista realmente. 
Ora, per ciò che riguarda il punto 1), Aristotele non ha mai ritenuto gli dei olimpici delle "false divinità", neppure dopo aver riconosciuto la divinità degli astri e del primo motore immobile. Per quanto riguarda il punto 2), il filosofo greco non si è mai domandato se un dio esista, con il rischio di non trovare sotto l'appellativo "dio" qualcosa di reale. Un dio, per Aristotele, come per tutti i Greci, è qualcosa di manifesto, anzi di massimamente manifesto, e perciò la sua esistenza è indubitabile. Il dubbio di Aristotele non è il dubbio scettico di colui che si pone di fronte alla possibilità di non trovare sotto l'appellativo "dio" qualcosa di reale. Il problema di Aristotele consiste piuttosto nel trovare una causa, una ragione che giustifichi la predicazione del termine "dio" agli enti cui questo attributo viene generalmente concesso, come i cieli, gli astri, il primo motore immobile, la parte noetica dell'anima umana o la natura nella sua interezza.

La questione aperta non è, pertanto, se la divinità esista, bensì in quale forma essa si realizzi nel modo più pieno. Decidere dell'esistenza di un dio, per Aristotele, si risolve nel verificare la pertinenza di una predicazione di eccellenza ad un soggetto, ossia nel provare se un ente essenzialmente meriti una certa attribuzione di eccellenza ${ }^{8}$. Aristotele non cerca se Dio, sostanza prima e unica del monoteismo cristiano, possa corrispondere ad una realtà, bensì se un dio, genere che si predica di altre sostanze, possa dirsi delle sostanze che vengono così chiamate. Il sentimento di Aristotele è che gli dei non siano dominio esclusivo della teologia tradizionale, e che scelgano molteplici espedienti per manifestarsi.

I cieli e gli astri sono considerati da Aristotele degli enti divini, benché non possano rappresentare la realtà suprema in quanto, come Aristotele spiega in Metafisica Lambda, essi sono mossi dai loro motori, e i motori immobili degli astri possiedono un grado di divinità superiore a quella dei corpi celesti. Aristotele può a buon diritto che la natura è un dio, al punto che egli usa talvolta l'endiadi "dio e la natura", in quanto vivente ed eterna. Tutte le specie viventi, in qualche misura, partecipano anch'esse del divino, perché sono eterne e si rendono eterne per mezzo della riproduzione. Meritano ancora la qualifica di divinità le sostanze immobili e fra le sostanze immobili l'attributo della divinità spetta al grado più elevato al Motore Immobile. Tra gli esseri viventi, è divino soprattutto l'uomo e nell'uomo è divina la parte più alta della sua anima, ossia l'intelletto (nous), che

${ }^{8}$ Cfr. Botter, 2005, pp. 15-32. 
Aristotele arriva a definire "massimamente congenere (suggenestatos) alla divinità" ossia della stessa stirpe della divinità, o anche "immagine (omoioma) di essa, e che di essa "partecipa (metechei)". A questo dio fa riferimento il frammento superstite del dialogo Sulla Preghiera.

Nel presente studio ci proponiamo di analizzare il frammento superstite dell'opera essoterica Peri Euches e di mostrare come esso possa essere posto in stretta relazione con un passo centrale del libro XII della Metafisica. Evidenzieremo come i due testi si completino l'un l'altro nel tentativo di cogliere la natura della divinità?

Il frammento Sulla Preghiera ${ }^{10}$

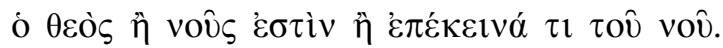

il dio è intelligenza o un ente che va al di là dell'intelligenza.

Un'opera di Aristotele sulla preghiera sarebbe altamente rivelatrice della sua mentalità religiosa. Ora, noi abbiamo alcuni testimoni autorevoli che precisamente ci informano sul fatto che Aristotele ha pubblicato un piccolo trattato intitolato Peri Euches oggi scomparso. L'esistenza del trattato è attestata da Diogene Laerzio nel suo catalogo con l'indicazione che quest'opera, probabilmente in forma di dialogo, non comportava che un solo libro (Peri Euches, alfa). Un altro catalogo che ricorda quest'opera del giovane Aristotele è quello della "Vita Hesychii" o "Vita Menagiana", in cui l'opera porta il n. 9. La fonte di questi cataloghi si pensa che sia Aristone di Ceo, uno dei successori di Aristotele alla testa del Liceo, come afferma Moraux, o Ermippo, come pensa Düring. In ogni caso gli elementi risalgono alla fine del III secolo, e ciò conferisce loro una autorità universalmente ammessa $^{11}$.

A noi non è pervenuto che un breve frammento dovuto a Simplicio, ed estratto dal suo commento al De Caelo. Giunto ad un passo importante $^{12}$, in cui lo Stagirita presenta i gradi di perfezione degli esseri,

${ }^{9}$ Cf. Plat., Rep. VII 532a-c. Vd. Allan 1952, p. 114; Organ 1962, pp. 303-305.

${ }^{10}$ Simplicio, In De Caelo 485, 19-22.

11 Vd. P. Moraux 1951, pp. 28 e 35. Nel catalogo di Diogene Laerzio l'opera porta il n. 14, ed. I. Düring 1957, p. 42.

${ }_{12}$ Arist., $D C$ II 12, 292b, 10-13. 
riservando un posto a parte alla perfezione ultima (ton ariston), lo scoliasta ricorda ciò che Aristotele scriveva al termine del suo dialogo Sulla Preghiera.

Riportiamo la testimonianza nel contesto in cui è inserita:

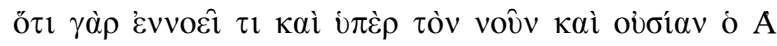

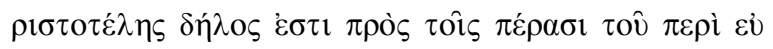

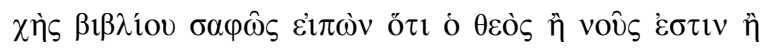

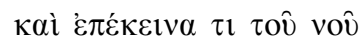

"il fatto che Aristotele pensi ci sia qualcosa al di là dell'intelletto e dell'essenza, appare chiaro quando, alla fine del libro Sulla Preghiera dice con una buona dose di saggezza che dio o è intelligenza o anche un ente che va al di là dell'intelligenza".

Noi analizzeremo tre sezioni di questo brano:

1. ennoei ti kai huper ton noun kai ten ousian;

2. ho theos nous estin;

3. ho theos estin epekeina ti tou nou.

1) è ricondotto indirettamente ad Aristotele, 2) e 3) gli sono espressamente attribuiti. La proposizione contrassegnata da 1) è di carattere prettamente neoplatonico. Nelle Enneadi troviamo: "il Bene è epekeina ousias ... kai epekeina nou"13; ed essendo epekeina ousias, è anche tou noein epekeina ${ }^{14}$.

2) Si incontrano delle difficoltà a trovare nei testi di Plotino l'affermazione che dio è intelletto. Si trova piuttosto l'affermazione inversa, secondo cui l'Intelletto è dio, è un dio multiplo: polus oun houtos ho theos epi tei psuchei ${ }^{15}$.

3) Si trovano in Plotino numerosi passi che pongono dio al di là dell'intelletto: dio va al di là della natura intelligibile e dell'essenza ${ }^{16}$;

\footnotetext{
${ }_{13}$ Plotino, Enneadi I 7, 1, 19-20, p. 119; V 1, 8, 6-8, p. 280.

14 Plotino, Enneadi V 6, 6, 29-30, p. 369.

${ }_{15}$ Plotino, Enneadi V 1, 8, 6-8, p. 280.

${ }_{16}$ Plotino, Enneadi I 1, 8, 8-10.
} 
dio è la causa e il principio dell'intelletto ${ }^{17}$. Il divino è posto da Plotino al di là dell'intelletto per il fatto che l'ente divino è superiore a ogni dualismo, mentre l'intelletto resta duplice, in quanto, anche quando pensa se stesso, raddoppia se stesso, perciò si rende duplice. A questi passi si possono aggiungere quelli in cui è detto che il Bene è al di là dell'Intelletto ${ }^{18}$.

Il frammento propriamente detto si riduce a 2) e a 3), i quali, nonostante l'ispirazione neoplatonica, hanno tutta l'apparenza di essere una citazione testuale.

Aggiungiamo, per completare il quadro generale, che tutta la tradizione medievale ha partecipato alla trasmissione di questo testo. Esso infatti è stato riprodotto in una Vita Latina di Aristotele tradotta dal greco, che porta la data dell'inizio del secolo XIII: Deus aut intellectus est aut aliquid ultra intellectum. Inoltre nel 1271 Guillaume de Mörbeke pubblicò, in versione latina, il commentario di Simplicio in cui il nostro frammento si trova inserito. Nel Medioevo nessuno dubitava dunque dell'origine aristotelica del documento in questione. Che valore ha ora tutto questo?

Innanzitutto ci sentiamo in dovere di fare una considerazione preliminare: un testo così breve, trasmesso, di fatto, da un solo autore, per quanto autorevole, non può aprire la via a delle affermazioni o a delle conclusioni definitive, ma piuttosto a delle scelte che risultano talvolta frutto di pure congetture. Tale avvertimento vale anche per le riflessioni che seguiranno.

A parte l'attestazione di un libro sulla preghiera, scritto da Aristotele, contenuta nei due cataloghi sopra citati, tutto riposa sulla fedeltà di Simplicio ${ }^{19}$.

Questa penuria di testimonianze ha spinto taluni a considerare l'autenticità del frammento molto dubbia. Ora, è vero che il testimone è unico, ma si tratta pur sempre di Simplicio, le cui attestazioni, in generale, meritano credibilità. Egli è stato definito da L. Robin: "commentateur d'une admirable érudition ... il nous a légué des scholies sur Aristote considérées comme une source de première valeur et on lui doit, pour une bonne part

\footnotetext{
${ }_{17}$ Plotino, Enneadi V 1, 11, 6-7.

18 Plotino, Enneadi VI 7.

${ }^{19}$ Aristotele non lascia infatti alcuna altra testimonianza sul tema dell'euche. Bonitz cita una quindicina di passi di riferimento, nei quali questo termine ricorre. Tuttavia in essi l'espressione è inserita in contesti in cui si tratta del culto ufficiale, e non ci sono pertanto di grande aiuto.
} 
(en raison de sa fréquentation de la bibliotéque de l'Académie) la transmission de nombreux fragments" 20 .

La difficoltà consiste nel fatto che abbiamo a che fare con un autore neoplatonico, il quale non dissimula la propria obbedienza a questa corrente filosofica. L'ispirazione neoplatonica è chiara. Nel brano che introduce il frammento, la dottrina espressa è aristotelica, ma non esclusivamente. È vero che Aristotele dice che il primo essere non ha bisogno di alcuna attività, ma anche per Plotino il primo principio non esercita alcuna praxis (Arnou 1921, pp. 21-22). Inoltre i due principi successivamente introdotti da Simplicio, Uno (Bontà) e Intelletto, sono ipostasi di Plotino. Tuttavia, non crediamo che dal neoplatonismo del testimone, si debba saltare troppo precipitosamente alla conclusione che le sue testimonianze sono contraffatte. Noi siamo dell'idea che ci siano dei termini ben precisi, riportati da Simplicio, che ci obbligano a procedere cautamente, prima di concludere che l'autore è mendace. Gli indizi a cui facciamo riferimento sono quattro:

1. il nome di Aristotele;

2. il titolo Peri Euches,

3. l'indicazione precisa: tou bibliou, "del libro", conforme alle notazioni delle liste antiche;

4. pros tois perasi, "alla fine del libro".

Se l'affermazione attribuita ad Aristotele fosse fittizia l'autore avrebbe avuto tutto l'interesse a rimanere nel vago. Perché rischiare di rapportarla ad un'opera determinata? Al contrario, Simplicio rende la testimonianza con una precisione assolutamente inabituale.

Oltre alle osservazioni di ordine esterno, se ne devono aggiungere altre di ordine interno:

il frammento concerne la natura del dio. Ora, studiando il brano, J. Bernays ha notato che la maggior parte dei trattati antichi Sulla Preghiera contengono una dissertazione sullo stesso tema: gli autori ritengono necessario risolvere il problema della natura di dio, al fine di risolvere tutte le questioni relative all'efficacia della preghiera ${ }^{21}$. Le riflessioni su un dio intelletto e al di là dell'intelletto stesso sarebbero dunque

${ }^{20}$ Robin, 1963, p. 10.

${ }^{21}$ Bernays, 1863, p. 122. 
perfettamente al loro posto in un Peri Euches, in cui Aristotele si sarebbe conformato alla tradizione.

La rispettosa esitazione che appare nell'alternativa "dio è questo, o anche quest'altro" corrisponde pienamente alla tradizione letteraria dell'innologia greca. E. Norden ricorda una incertezza analoga nella maggior parte delle preghiere antiche, in cui essa veniva espressa attraverso una formula disgiuntiva ${ }^{22}$.

Alcuni di questi testi risalgono al V secolo, e si tratta, in particolare, delle riflessioni dei tragici. L'incertezza relativa alla natura divina si traduce in espressioni come hostis ei nell'Agamenonne di Eschilo: Zeus hostis pot'estin ${ }^{23}$; nell'Eracle di Euripide: Zeus d'hostis ho Zeus ${ }^{24}$; oppure hopoteron onima, "qualunque sia il tuo nome" nelle Bacchanti di Euripide: Demeter thea ge d'estin onoma d'hopoteron boulei kalei ${ }^{25}$. Ora, Aristotele nutrì sempre un grande interesse per gli autori tragici. È sufficiente a confermarlo il numero di riferimenti espliciti ad Euripide e a Sofocle, riportato da Bonitz ${ }^{26}$. Che difficoltà ci sarebbe a credere che, nella conclusione di uno scritto sulla preghiera, Aristotele si ispiri ad essi? Anche solo alla luce di queste brevi considerazioni, le ragioni che spingono a dubitare di questo documento ci paiono deboli.

\section{Le interpretazioni tradizionali}

II frammento Sulla Preghiera, crux interpretum, è verosimilmente protoaristotelico. La seconda parte di esso potrebbe riflettere il pensiero di Platone, o per lo meno alludere ad una dottrina, la cui paternità è da attribuirsi a Platone. Da questa proposta ermeneutica si possono trarre due ipotesi:

l'autore espone il pensiero di Platone e non il proprio;

22 Norden, 1913, p. 143-257.

${ }^{23}$ Eschilo, Agamennone 160.

${ }^{24}$ Euripide, Eracle 1263.

25 Euripide, Baccanti 275-276. Inoltre, formule di questo tipo si trovano anche in Platone, precisamente nel Cratilo 400e: "invocarli (gli dei) come è nostra consuetudine nelle preghiere e, chiunque siano e da qualunque parte provengano, con quei nomi con cui desiderano essere chiamati"; nel Filebo 12c: "II mio timore di sempre, di fronte ai nomi degli dei [...] va oltre la più grande paura", 30d: "e allora tu dirai che nella natura di Zeus si trovano un'anima regale ed una intelligenza regale a motivo del potere della causa..."; nel Fedro 246d: "il termine immortale non può essere spiegato sulla base di un solo discorso razionale; ma, senza conoscerlo ed intenderlo in modo adeguato, noi ci figuriamo un dio, un essere vivente ed immortale, che ha un'anima ed un corpo eternamente connaturati".

${ }^{26}$ Bonitz: riferimenti ad Euripide: B p 300a 10-59; riferimenti a Sofocle: B p 689a47- 689bl3. 
l'autore esprime la propria idea personale, la quale risente dell'influenza della teologia delle Leggi e del Timeo.

La prima ipotesi (a) non ci pare, almeno a prima vista, inverosimile e può ben essere in armonia con lo stile di un breve trattato, che doveva rivestire la forma del dialogo. Questa congettura è stata avanzata per la prima volta da Bignone. Giunto a questo punto dello sviluppo dottrinale (pros tois perasi), è possibile che Aristotele riassuma la situazione dialettica prima di concludere e, forse, di ribadire la propria convinzione personale. In altri termini, si avrebbe una situazione di questo tipo: dio è, in conclusione, un nous, o ancora, come direbbe Platone (sottinteso) un al di là del nous.

A questo punto l'interrogativo che si pone è di sapere se c'è nell'opera di Platone qualche elemento che ci autorizzi ad attribuire questa soluzione al filosofo ateniese. Precisamente si incontra il termine epekeina in Platone una sola volta, nel passo famoso della Repubblica: "E cosi anche ai conoscibili dirai che proviene dal Bene non solo l'essere conosciuti, ma anche l'essere e l'essenza provengono loro da questo, pur non essendo il Bene l'essere, ma ancora al di sopra dell'essere (epekeina tes ousias), superiore ad essa in dignità e potere"27.

$\mathrm{Si}$ può ben dire che ci troviamo in un contesto teologico, all'interno del quale l'intelletto è trasceso dal bene. Quando Aristotele, nel De Anima, riporta la dottrina corrispondente, ci dice chiaramente che è frutto della filosofia del suo Maestro: "l'animale in sé proviene dall'Idea stessa dell'Uno"28.

Nel Filebo, dopo aver elencato i caratteri del Bene, bellezza, proporzione, verità, Platone offre una specie di valutazione gerarchica, che vede l'intelletto solo al terzo posto (triton ... noun) ${ }^{29}$. Non è dunque escluso a priori che Aristotele abbia potuto cogliere, nel Maestro, oltre all'idea di una divinità la cui superiorità supera ogni capacità di intendimento umano, quella di una profondità, dal punto di vista ontologico, che situa la divinità al di là di ogni definizione, al di là anche della stessa nozione di nous.

Non nascondiamo che la glossa di Simplicio: hoti gar ennoei ti kai huper ton noun kai ten ousian ho Aristoteles, ci ricorda il Platone della Repubblica: epekeina tes ousias.

Resta tuttavia difficile riferire l'epekeina del Sulla Preghiera alla dottrina platonica dell'Uno-Bene. Troppe ombre aleggiano su questa dottrina

\footnotetext{
27 Plat., Repubblica VI 509b.

${ }^{28}$ Arist., DA I 2, 404b20.

29 Plat., Filebo 66b.
} 
nei dialoghi platonici. Inoltre Simplicio riconduce la paternità di questa definizione al solo Aristotele, e in una forma che assomiglia fortemente ad una citazione testuale. Inoltre, è la stessa definizione di "intelletto" che varia nei due filosofi: Aristotele definisce il nous una facoltà o una sostanza; al contrario, nella visione platonica, l'intelletto è soprattutto atto del sapere, come appare evidente nella Repubblica ${ }^{30}$, nel Timeo $^{31}$ e, in particolare, nel Fedro: "La ragione di un dio si nutre di intelligenza e di scienza pura"32. Ora, nel frammento di Aristotele l'intelligenza sembra essere una realtà sostanziale.

La seconda ipotesi (b) ci sembra che raggiunga un grado superiore di verosimiglianza. Questa è la posizione tenuta da P. Friedlander, J. Pépin, e R.A. Gauthier e J.Y. Jolif: "Au moment où il écrivait son dialogue "De la prière” le jeune Aristote avait, il est vrai, retenu l'idée platonicienne d'un Dieu qui serait au-delà de l'intellect"33.

Recentemente questa interpretazione è stata accolta da Aryeh Kosman, il quale commentando l'espressione ei de mallon di Metafisica XII 7, 1072b24-25, scrive: “Aristotle's “more” may recall Plato's description of the good as "beyond substance - epekeina tes ousias" (Republic 509b9). Simplicius clearly thought something of the sort, for he supports his claim that Aristotle had in mind something beyond thought by quoting from the lost On Prayer Aristotle's assertion that "the divine is either thought or something beyond thought (in De Caelo 485.19-20; cfr. EE VII 14, 1248a27). The view that Aristotle offers, it seems, is not simply that God thinks as we do, only all the time rather than merely some of the time; it is rather that God engages in an activity that is like thinking, but something more" 34 .

Nuyens ${ }^{35}$ legge nel frammento la netta affermazione che la divinità è essenzialmente intelletto: "Le point important est que, pour Aristote, l'essence de la divinité, c'est d'être intelligence”.

Secondo J. Bernays, seguito in questa interpretazione da H. Cherniss, E. Berti, e des Places ${ }^{36}$, Aristotele, dopo aver fondato la propria concezione della divinità sulla nozione di nous, tende a precisare che l'intelletto di dio, a

\footnotetext{
${ }^{30}$ Plat., Repubblica VI 18, 506c7; IV 8, 431a5.

31 Plat., Timeo 51d3.

${ }^{32}$ Plat. Fedro $247 \mathrm{~d} 2$.

${ }^{33}$ Friedlander, 1928, pp. 67 sg.; Pépin, 1968, pp. 68 sg.; Gauthier-Jolif, 1970 II 2, p. 856.

${ }^{34}$ Kosman 2000, pp. 310-311.

35 Nuyens 1948, p. 133.

36 Bernays, 1863, p. 123; Cherniss, 1955, p. 592; Berti, 1997, p. 386; des Places, 1969, p. 256.
} 
cui è riferita la prima parte del testo, è al di là dell'intelletto umano, privo dei limiti, delle debolezze, delle contraddizioni che appesantiscono il dinamismo intellettuale dell'uomo. Nell'intento di preservare inviolata la purezza del divino, lo Stagirita porrebbe un abisso fra intelletto umano e intelletto divino. Questa interpretazione è possibile, ma crediamo necessario sfumarne i contorni perché, se anche esprimesse il pensiero autentico dello Stagirita, Aristotele non ci presenta l'idea in modo così chiaro. L'argomento di Bernays si può suddividere in questo modo:

1. dio è nous,

2. dio è qualcosa che va al di là del nous umano.

Ora, è facile mostrare che le due proposizioni appartengono entrambe alla teologia aristotelica delle opere della maturità:

1) che la divinità sia nous è detto in De Partibus Animalium, Etica Nicomachea, Metafisica, De Anima, De Generatione Animalium e già in un frammento del Protrettico 37.

Dire che l'intelletto dell'uomo è divino non impedisce di pensare che debba essere inferiore ad un dio. È questo ciò che lasciano intendere molti passi di Metafisica, ma soprattutto l'Etica Eudemia ${ }^{38}$.

L'opera di Aristotele, compresa l'opera a noi pervenuta solo frammentariamente, offre dunque un solido appoggio alle due proposizioni di Bernays.

Tuttavia, l'interpretazione di Bernays non ci persuade interamente: innanzitutto, crediamo essa contenga una imprecisione dal punto di vista grammaticale. Quando il logico Aristotele utilizza lo stesso termine nous nella prima e nella seconda parte della disgiuntiva, non può che paragonare due termini aventi lo stesso senso, ma anche la stessa estensione. Il termine nous, senz'altra precisazione, può ricoprire una estensione differente nel primo e nel secondo membro della frase? Per poter accettare questa interpretazione, dovremmo poter leggere qualcosa del tipo: l'intelletto di dio, a cui è riferita la

\footnotetext{
${ }^{37}$ Arist., PA IV 10, 686a29; EN I 4, 1096a24-25; Metaph. XII 7, 1072b27; Metaph. XII 9, 1074b21-22; Metaph. XII 7, 1072b23; cfr. 9, 1074bl5-16; DA I 4, 408b29; GA II 3, 736b28; 737a9-10; EN X 7, 1177al316; EN X7,1177b30; EN X 8, 1179a26-27; Protrettico fr. 10c Ross, p. 42 = Giambico, Protreptico 8.

${ }^{38}$ Arist., Metaph. XII 7, 1072b24-26; Metaph. XII 9, 1074b31-34; Metaph. XII 9 1075a5-9; EE VIII 2, 1248a24-32.
} 
prima parte del testo, è al di là dell'intelletto umano. Si passa dal nous, in generale, al nous umano.

Ciononostante il riferimento che Bernays fa alla Metafisica ci pare estremamente interessante e ce ne serviremo per illustrare la nostra ipotesi interpretativa, secondo la quale il Peri Euches e la Metafisica si muovono nella stessa direzione.

J. Pépin ${ }^{39}$ legge nel nostro frammento una confessione di fede cosmica. E non è il solo. In seguito al lavoro di Fèstugière sul "Dieu cosmique" 40 molti studiosi credono che la presenza di un dio cosmico sia stata particolarmente cara ad Aristotele all'epoca dei trattati essoterici.

Il frammento in questione tratta della presenza di questa divinità? La questione è duplice:

Il Dialogo sulla preghiera tratta della "preghiera cosmica"?

La formula incriminata rivela la presenza di una concezione di questo tipo in Aristotele?

Alla luce di un minuscolo frammento, e in mancanza di testimonianze più generose, la cosa non ci sembra facilmente sostenibile, a meno che non ci si lasci andare al di là delle premesse.

L'autore fonda la propria ipotesi su di un parallelismo, che egli trova sorprendente, tra il nostro testo e il frammento 26 Ross del De Philosophia, in cui Cicerone attribuisce ad Aristotele fedeltà ad una triplice divinità: la mens, il mundus, e un terzo principio: alium quemdam: "Aristotelesque in tertio de philosophia libro multa turbat a magistro uno Platone dissentiens. Modo enim menti tribuit omnem divinitatem, modo mundum ipsum deum dicit esse, modo alium quendam praeficit mundo; Aristotele nel terzo libro del suo dialogo Sulla Filosofia espone in modo incoerente molte idee, venendo in disaccordo su un punto col maestro Platone. Ora attribuisce ogni divinità all'intelletto, ora sostiene che il mondo stesso è un dio, ora pone un altro ente a capo del mondo"

Secondo lo studioso francese, la mens non è altro che l'intelletto del mondo, il mondo non pone difficoltà nel quadro della religione cosmica, resta questo misterioso "alius quidam", che ha il compito di sostenere il movimento generale dell'universo. A questo fenomeno Pépin riferisce l'epekeina del Trattato sulla Preghiera.

\footnotetext{
39 Pépin, 1968.

40 Fèstugière, 1949.
} 
Ora, questa ipotesi, per quanto seducente e sottile, ci pare difficile da sostenere. Essa risulterebbe plausibile solo se venissero confermati almeno tre elementi:

a) che la mens nel frammento 26 altro non sia che l'intelligenza del mondo;

b) che l'alius quidam si presenti come un al di là di questa mente;

c) che l'epekeina ti tou nou del frammento Sulla Preghiera possa essere tradotto "un al di là dell'intelligenza del mondo".

Ora: a) se la nozione di intelletto cosmico, anima del mondo, si incontra effettivamente in Platone, noi pensiamo che non sia facile rintracciarla nel De Philosophia, né nel De Caelo, né in alcuna altra opera di Aristotele.

b) non solo alius quidam non è definito "al di là della mente", ma non si può neppure affermare con certezza che sia epekeina di qualcosa nell'elenco dei diversi enti divini ricordati da Cicerone e attribuiti ad Aristotele.

c) non troviamo alcun elemento plausibile che autorizzi Pépin a tradurre in un certo modo, per giunta molto specifico, questo passo, a preferenza di altre traduzioni.

Siamo pertanto spinti nuovamente al testo greco, così come esso si presenta alla prima lettura, e nella forma in cui la tradizione ce lo ha consegnato. Forse l'ipotesi più verosimile è anche la più semplice.

\section{Interpretazione personale}

Non possiamo negare che il carattere divino dell'intelligenza sia una costante della metafisica aristotelica. Il nous, in generale, è nel Corpus aristotelicum la facoltà di cogliere l'intelligibile e ciò che c'è di più divino nell'uomo ${ }^{41}$. È dunque una relazione molto intima quella che lo Stagirita instaura fra l'intelletto e un dio. Inoltre in un'epoca approssimativamente contemporanea all'apparizione del nostro dialogo, nel Protreptico il filosofo sostiene esplicitamente che è in grazia dell'intelletto che l'uomo assomiglia a un dio. Perciò, a nostro avviso, l'affermazione "dio è

41 Vd. Arist., EN X 7, 1177a13. 
intelligenza" non si rivela significativamente diversa dall'espressione "l'intelligenza è divina", intendendo con ciò l'intelligenza umana, affermazione questa che ricorre frequentemente nel Corpus aristotelicum.

Ciò che resta aporetico è la seconda parte della proposizione: epekeina ti tou nou. Il termine epekeina, secondo Bonitz, è usato solo sei volte da Aristotele e mai, se si esclude questo frammento, in un contesto metafisico o teologico. A nostro avviso, questo non indebolisce la veridicità della testimonianza, in quanto la medesima osservazione si potrebbe fare per Platone, ma nessuno dubita della validità di Repubblica VI 509b. Non possiamo però nascondere che l'espressione è estranea al formulario abituale di Aristotele, ed è nettamente tendente al neoplatonismo. Per questo motivo l'ermeneutica non può che essere molto delicata.

Noi riteniamo interessante la suggestione offerta da Bernays in merito ad una relazione fra il frammento del Peri Euches e il libro XII della Metafisica, pur non trattandosi di una identità. Facciamo notare che nel frammento non siamo in presenza della noesis noeseos, bensì del nous, e le due facoltà non sono identiche. Nello stesso libro XII della Metafisica non sarà immediata la caratterizzazione del Motore Immobile come noesis, bensì frutto di un articolato ragionamento, che si snoda attraverso Lambda 6, 7 e 9, e vede arricchirsi di caratteristiche sempre più precise e determinate il concetto di principio primo: da noeton, a nous, a noesis ${ }^{42}$. Metafisica Lamda 7 è il testo che maggiormente si avvicina al frammento del dialogo essoterico e indica la direzione della riflessione aristotelica. In questo testo, Aristotele si riferisce alla natura divina come ad un nous, precisamente un nous che pensa se stesso cogliendosi come intelligibile (hauton de noei ho nous kata metalepsin tou noetou $)^{43}$. In Lambda 9 un passo ulteriore verrà compiuto, in quanto è il "pensiero di pensiero" (noesis) ad agire ${ }^{44}$. La necessità di questo ulteriore passaggio è dovuta alla differenza che caratterizza il nous e la noesis. Il nous è la facoltà di intelligere, quindi, per definizione, non è necessariamente in atto; la noesis è atto di intellezione, l'azione di fare qualcosa, intelligenza in atto. È l'inizio di Lambda 9 a rivelare questa difficoltà: ciò che abbiamo detto del nous solleva una aporia, e questa concerne il carattere potenziale del nous. Il passaggio a Lambda 9 è preparato in Lambda 7, di cui forniamo una breve analisi per mostrare le similitudini con il Peri Euches, in particolare su

\footnotetext{
42 In particolare, il passaggio da nous a noesis avviene solo in Lambda 9. Cfr. Botter, 2005, pp. 173-217.

${ }^{43}$ Arist., Metaph. XII 7, $1072 \mathrm{~b} 20$.

${ }^{44}$ Arist., Metaph. XII 9, $1074 \mathrm{~b} 34$.
} 
due punti: la costante analogia con la condizione dell'intelletto umano e la possibilità che il divino sia superiore alla condizione umana; quindi l'esitazione a dire l'ultima parola sulla natura della divinità.

Uno dei problemi lasciati aperti dalla Metafisica, malgrado il desiderio dell'autore di risolverlo, concerne la natura del principio. Essa non può essere colta in modo diretto, ma solo per analogia con la condizione umana. Il primo passo nel corso dell'argomentazione sulla natura del divino consiste nell'illustrare come esso muova il cielo. Alle linee 1072a26-b4 Aristotele asserisce che il principio primo muove hos eromenon, ossia in quanto oggetto di intelligenza e di desiderio: "muovono in questo modo l'oggetto del desiderio e l'oggetto dell'intelligenza”, ossia muovono senza essere mossi, quindi il principio, conclude il filosofo, "muove come oggetto di amore, mentre le altre cose producono movimento perché sono esse stesse mosse"45. Da questa conclusione sembra chiaro che il motore muova come causa finale, ma la tesi pare in contrasto con quanto risulta da Lambda 6, di cui Lambda 7 richiama il traguardo raggiunto, e in cui il motore del cielo è introdotto in questo modo: "ma invero, se c'è alcunché capace di muovere e di fare

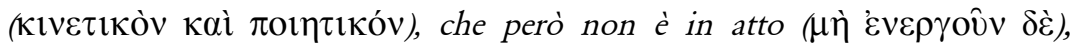
non ci sarà movimento, poiché è possibile che ciò che ha potenza non sia in

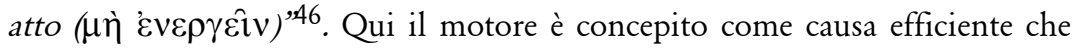
agisce in atto ${ }^{47}$. La ragione di questa necessità è resa esplicita nel passo successivo in cui lo Stagirita critica la pretesa delle Idee platoniche a speigare il movimento: le Forme ideali, pur essendo eterne e immobili, non possono spiegare il movimento, in quanto non contengono alcun principio capace di produrre mutamento e, se anche lo contenessero, come Platone pretende, questo principio non è attualmente operante. Quindi Aristotele descrive le caratteristiche che il principio deve possedere per poter muovere restando immobile. Alle linee 1072a26-b4 il filosofo focalizza l'attenzione sull'oggetto della facoltà di desiderio: dapprima identifica il desiderabile (ỏ $\rho \varepsilon \kappa \tau o ́ v)$ con

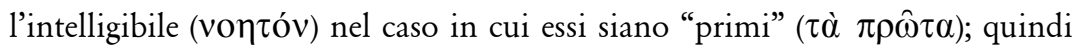
identifica il desiderabile intelligibile al bello e/o al bene, ossia all'ottimo. Non abbiamo due generi di Motore immobile, il desiderabile (ò $\rho \kappa \tau$ óv) e

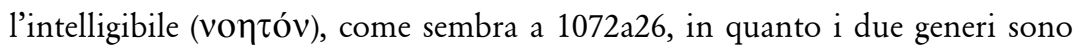

\footnotetext{
${ }^{45}$ Arist., Metaph. XII 7, 1072a26-27; b3-4.

${ }^{46}$ Arist., Metaph. XII 6, 1071b12-14.

47 I termini kinetikon kai poietickon in cui il suffisso -tikon indica la capacità di fare qualcosa sono normalmente usati da Aristotele per indicare la causa motrice. Cfr. GC I 7, 432b13-14.
} 
il medesimo, nel caso in cui certe condizioni siano soddisfatte: $\tau$ ov́ $\tau \omega \nu \tau \grave{\alpha} \pi \rho \hat{\omega} \tau \alpha \tau \grave{\alpha} \alpha \hat{u} \tau \alpha^{48}$.

La sezione 1072b14-30, quella che maggiormente ci interessa per la relazione al frammento del Peri Euches, affronta il tema della $\delta 1 \alpha \gamma \omega \gamma \eta ́$ del principio primo, ossia la sua "occupazione", il suo "modo di vivere". Il primo motore non è, infatti, pura esistenza, bensì vita. Ciò appare

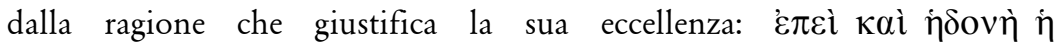

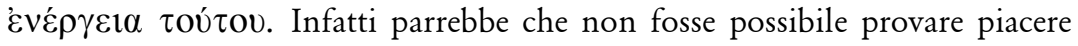
in assenza di attività. Ma niente finora ci ha preparato all'idea che

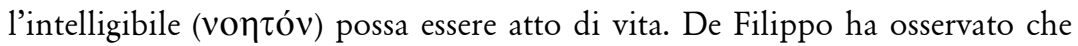
se l'identificazione del principio, finora solo noeton, con l'intelletto, nous, non è stata resa in modo esplicito, nondimeno essa consegue da quanto è stato detto, in quanto un intelligibile (noeton) non può esistere in atto se non viene pensato da un intelletto che pensa ${ }^{49}$. Perciò se Aristotele afferma che il principio è atto e heromenon intelligibile, è chiaro che esso è intelletto, o meglio intelligere. Ma questa elegante ricostruzione non risulta convincente sotto tutti i punti di vista. Infatti, Aristotele non sottintende che un intelligibile possa essere in atto solo se è oggetto di un intelletto pensante, bensì egli ritiene che questo intelligibile formi una unità con l'intelletto stesso. Noi sappiamo da 1072a27-b4 che il primo intelligibile è, per essenza, in atto, ossia è la sostanza la cui attualità non richiede di essere ulteriormente garantita. Insomma, il primo intelligibile non è in atto perché è pensato da un intelletto in atto; bensì è esso stesso un intelletto in atto. Tale lettura non elimina il carattere ellittico dell'argomentazione di Aristotele. Come le linee 1072a27-b4 possono essere comprese solo alla luce della psicologia del $D e$ Anima, così la sezione 1072b14-30, può essere compresa solo facendo riferimento all'intelletto umano. Riproduciamo il testo suddividendolo in sezioni in modo che emerga più chiaramente l'argomentazione aristotelica:

$$
1=1072 \mathrm{~b} 14-16
$$

E il suo modo di vivere è il più eccellente: è quel modo di vivere che a noi è concesso solo per breve tempo (e in quello stato egli è sempre; a noi invece questo è impossibile) poiché l'atto del suo vivere è piacere.

\footnotetext{
48 L'identità del primo desiderabile con il primo intelligibile è fondata sulla dottrina esposta in De Anima III 10. Per una argomentazione più dettagliata, vd. Botter 2005, pp. 185-195.

49 De Filippo, 1994, pp. 303-409.
} 
$2=1072 \mathrm{bl} 6-18$

E per questo veglia, sensazioni e conoscenza sono in sommo grado piacevoli, e, in virtù di queste, anche speranze e ricordi.

$3=1072 \mathrm{~b} 18-19$

il pensiero per se stesso è di ciò che è per sé massimamente eccellente, e il migliore è di tanto superiore quanto più il pensiero è tale.

4= 1072b19-23

L'intelligenza pensa se stessa in seguito al coglimento dell'intelligibile (cogliendosi come intelligibile), infatti diventa intelligibile toccando (intuendo) e pensando (sé), cosicché intelligenza e intelligibile coincidono (il nous infatti è ciò che è capace di cogliere l'intelligibile e la sostanza, è in atto quando li possiede),

$5=1072 \mathrm{~b} 23-24$

Cosicché ekeino (hautos noei) più ancora che toutou (capacità di cogliere l'intelligibile e la sostanza, to dektikon tou noetou kai tes ousias) è ciò che il nous ha di divino, e la theoria l'attività più piacevole e la migliore.

$$
6=1072 \mathrm{~b} 24-26
$$

Se dunque in questa felice condizione in cui noi ci troviamo talvolta un dio si trova sempre, è meraviglioso se si trova in una condizione superiore, è ancora più meraviglioso. $E$ in questa condizione effettivamente si trova.

\section{$7=1072 \mathrm{~b} 26-30$}

Ed egli è anche vita, perché l'attività dell'intelligenza è vita, ed egli è appunto quell'attività. E la sua attività, che sussiste di per sé, è vita ottima ed eterna. Diciamo d'altro canto che un dio è un vivente eterno ed ottimo, cosicché ad un dio appartiene una vita perennemente continua ed eterna: questo è dunque un dio.

Nella sezione 1, Aristotele introduce due elementi: dapprima l'eccellenza dello stato del principio, al quale la condizione migliore di cui l'uomo può godere offre un termine di paragone (hoia he ariste); in seguito la durata di questo stato: non è limitato ad una piccola porzione di tempo, 
mikron chronon, come per l'uomo. Il brano ritorna quindi su ciascuna di queste due determinazioni. Fino a questo momento, la differenza fra l'uomo e il principio pare fondata sulla semplice durata temporale, non è escluso, infatti, che l'attività del principio sia identica, dal punto di vista dell'eccellenza, alla migliore fra le attività umane. Tuttavia il termine hoia lascia presagire che sia solo simile. Del resto al punto 6 la possibilità di una superiorità dell'attività divina su quella umana, da un punto di vista differente rispetto alla pura temporalità, è esplicitamente evocata: ei de mallon $^{50}$, eti thaumasioteron. Ora, la durata non è più menzionata prima della conclusione, essa è fatta oggetto di una semplice asserzione, in quanto non risulta di natura problematica. L'eccellenza, al contrario, è resa oggetto di una analisi esplicita.

Per quanto il termine theoria non sia immediatamente sostituibile a noesis, noein, cosa che invece in questo specifico caso noi ammetteremo, crediamo che la conclusione di Aristotele sia che la migliore attività è quella di natura noetica (5), in quanto he theoria, se riferita all'attività del principio, equivale a hautos noei. È possibile trovare nel testo in questione un argomento che confermi questa ipotesi, e conduca da 2 a 5? Se un argomento esiste, il concetto di piacere deve fungere da termine medio.

A questo riguardo, è possibile rendere una duplice interpretazione del testo. Una prima esegesi considera il "piacere" come premessa della dimostrazione aristotelica; una seconda interpretazione assume, invece, il "piacere" come ultima tappa dell'argomentazione che conclude al "pensare" come all'attività propria dell'ente supremo. Consideriamole entrambe. La prima è la seguente:

A 1 la diagoge è detta ariste perché il suo atto è piacere, epei kai hedone.

A 5 i due predicati hediston kai ariston sono coordinati, ma l'argomento è costruito come se l'eccellenza derivasse dal piacere, piuttosto che l'inverso. Da ciò è possibile concludere, tenuto conto del capitolo $6 \mathrm{di}$ Lambda, che l'attività piacevole è attività di una intelligenza?

Aristotele sembra dare per scontato che l'attività del Motore Immobile sia piacere, anzi usa questa premessa per concludere che, essendo

${ }^{50}$ Mallon eu in opposizione a houtos eu. 
per noi il pensiero l'attività più piacevole, di conseguenza l'attività del Motore Immobile sarà pensiero.

Ora, la premessa secondo cui l'attività del principio primo è piacere, non è una presupposizione pura e semplice. Le ragioni vanno cercate nell'Etica a Nicomaco ${ }^{51}$, in cui appare: touto d'esti hedone in cui touto = energeia. L'atto del principio primo è piacere, perché l'atto, in quanto tale, è piacere. Questo tipo di rinvio, peraltro, è moneta corrente in Lambda: spesso Aristotele suppone che la giustificazione delle premesse sia nota. Dalla premessa ora enunciata, è possibile procedere in questo modo:

a) l'atto in quanto tale è piacere ${ }^{52}$;

b) il più grande piacere che noi conosciamo è quello dell'attività di pensiero (2: noesis, 5: theoria), perché fra tutte le funzioni pratiche possiede un grado minimo di potenzialità ${ }^{53}$;

c) l'attività del principio primo (6), nella misura in cui è atto, è piacere;

d) la sua attività è dunque quella più piacevole, perciò quella che possiede il grado minimo di potenzialità, quindi è una attività di pensiero.

La seconda possibilità di lettura consiste nel porre il "piacere" come conclusione del fatto che l'attività della divinità è "pensare". Il punto di partenza è, ancora una volta, 1: "Il suo modo di vivere è il più eccellente [...] poiché l'atto del suo vivere è piacere". A partire da ciò si può concludere che l'attività piacevole è il "pensare"? Questo esito è consentito, a nostro avviso, $\mathrm{da}$ tre considerazioni, che trovano origine nel libro $\mathrm{X}$ dell'Etica Nicomachea:

1 L'attività di pensiero si basa su di una virtù perfetta che non incontra ostacoli nella sua realizzazione ${ }^{54}$;

2 - essa gode della sua maestria e perfezione ${ }^{55}$;

3 -il pensare non ha bisogno di mezzi materiali per realizzarsi ${ }^{56}$.

\footnotetext{
51 Arist., EN VII 13, 1153b12.

${ }^{52}$ Arist., EN VII 13, $1153 \mathrm{~b} 12$.

${ }^{53}$ Cf. Laks, 2000, pp. 233-234.

${ }^{54}$ Arist., EN X 7, 1177a18-21; cf. Metaph. XII 7, 1072b18-19.

${ }^{55}$ Arist., EN X, 1177a27 e $1177 \mathrm{~b} 1$.

${ }^{56}$ Arist., DA III 8, 431b21; III 4, 429a19-19; III 4, 429a27.
} 
Perciò l'attività del pensare è la più piacevole e la migliore (5). E se l'attività del pensiero umano nella sua realizzazione è così caratterizzata, la condizione del dio sarà addirittura superiore a quella dell'uomo. Infatti il dio ha il più alto grado di essere ${ }^{57}$ e l'oggetto di attività, per il dio, è il più bello e perfetto $^{58}$.

Nella seconda sezione, Aristotele, di tutte le funzioni pratiche, prende in considerazione le tre più importanti, in quanto esse procurano, se non il piacere più grande, in quanto non tutte sono piacevoli allo stesso modo, almeno un piacere piuttosto grande (hediston). La gerarchia è determinata dal grado di potenzialità insito in ciascuna di queste facoltà, il quale risulta minimo nel caso del comprendere. Esso, infatti, è la sola facoltà in grado di produrre il suo proprio oggetto. Se queste funzioni sono piacevoli, ciò è dovuto al fatto che sono attività (energeiai). Il superlativo in questione è da una parte assoluto, dall'altra relativo. È assoluto in quanto le tre funzioni menzionate formano una unità in opposizione ai piaceri procurati dalle funzioni psichiche inferiori, come la nutrizione; è relativo, perché, tra le tre funzioni menzionate, una eccelle sulle altre.

Nella terza sezione, il superlativo hediston indica il grado supremo di un piacere che, pure, ammette dei gradi. Aristotele concluderà infatti che he theoria he hediston kai ariston (5) segnando, in questo modo, l'apice della scala, ossia dio; infatti, l'ente la cui natura fa tutt'uno con l'attività qui descritta è dio. Il ragionamento seguito dallo Stagirita è il seguente: se ciascuna di queste tre facoltà ha un oggetto proprio (ciò resta implicito), il migliore oggetto è quello del pensiero. Il termine noeton non è menzionato, ma è di esso che si tratta nella specificazione tou ... aristou (3). L'argomentazione della sezione 3 si può suddividere in due parti:

- il pensiero, in quanto tale, pensa il migliore (he de noesis he kath'hauten tou kath'hauto ariston);

- il migliore è tanto superiore, quanto più il pensiero è tale (kai he malista tou malista).

L'idea che esistano dei gradi di pensiero lascia aperta la possibilità che per Aristotele esista una differenza sostanziale tra attività umana e attività divina

${ }^{57}$ Arist., Metaph. XII 7, 1972b14-16.

58 Arist., Metaph. XII 9, 1974b15-1075a10. 
e conferma che la scelta compiuta da Aristotele di adottare il termine "divino" è, precisamente, dovuta alla necessità di indicare, all'interno di una modalità dell'essere, l'attività del grado più eccellente.

La sezione quarta (1072b20) specifica la natura del migliore, grazie all'identificazione dell'intelligibile di 1072a27-b4 con l'intelletto stesso. In un certo senso, appare qui, per la prima volta, l'idea che il Motore Immobile sia pensiero di pensiero, idea che verrà sviluppata in Lambda 9. Facciamo notare che l'argomento esposto da 2 a 5 si riferisce al comprendere in generale, quindi vale per ogni comprendere, tanto per il pensiero umano, quanto per il pensiero divino.

Anche l'affermazione della sezione 5 concerne l'intelletto in generale: ciò che è divino, ossia eccellente, nell'attività dell'intelletto, è il fatto che l'intelletto pensa se stesso pensante.

La sezione setsa rimanda specificamente al principio primo, che ora viene designato "dio". Avendo stabilito a 1 che l'attività del principio primo è piacere, ed avendo affermato nell'Etica Nicomachea che gli dei godono del piacere e della felicità ${ }^{59}$, Aristotele può ora affermare che il principio primo è un dio. Esso è per eccellenza un dio: dall'eccellenza dell'intelletto in generale (5) Aristotele ha compiuto un ulteriore raffinamento: il divino intelligere è nella sua sostanza propria superiore all'umano intelligere, benché sia difficile, sulla base delle indicazioni offerte fino a questo momento, rendere in modo più preciso in cosa consiste la sostanziale superiorità del divino comprendere, rispetto al comprendere umano. È vero, infatti, che entrambe pensano il migliore. L'argomentazione su questo punto importante si arricchirà di elementi preziosi al capitolo 9 del libro Lambda.

Nell'ultima sezione il filosofo presenta un argomento a favore dell'identificazione di un dio con la vita.

Da parte nostra non siamo convinti che questa argomentazione sia la conclusione di Aristotele sulla natura del dio. Non è per unanime accordo, infatti, che le ultime linee, 1072b28-29, sono ritenute essere la conclusione di Aristotele. Per segnalare questa incongruenza nella traduzione della sezione 7 abbiamo marcato "d'altro canto", che traduce letteralmente la particella $\delta \grave{\varepsilon}$ nel testo greco. La versione tradizionale legge, invece, $\delta \grave{\eta}$, ossia "noi affermiamo dunque che ...”. Questa lezione non è unanime dei manoscritti, bensì è uniforme all'interpretazione di Temistio ed accettata dalla maggior

${ }^{59}$ Arist., EN X 8, 1178b8-9 e b24. 
parte degli interpreti, da Bonitz, a Jaeger a Ross. Noi crediamo, invece, che il filosofo si limiti a rievocare ciò che comunemente si dice di un dio e che manifesti un segno di modestia, un modo per dichiarare la debolezza umana nel momento in cui voglia esprimere la natura reale di un dio.

Infine, torniamo alla nostra interpretazione del frammento Sulla Preghiera. Essa si caratterizza per il fatto di attribuire al termine nous il senso di "intelligenza umana", in entrambe i membri del frammento, in quanto crediamo che Aristotele si serva di una analogia con l'attività umana, come nel caso di Metafisica Lambda: "un dio è intelligenza", che è come dire "l'intelligenza è una facoltà divina" e al di là dell'intelligenza umana, ossia l'ente o gli enti supremi hanno una natura che sfugge alla comprensione umana, e può essere colta dall'uomo solo attraverso una sorta di analogia con ciò che egli meglio conosce: la sua propria intelligenza. Crediamo che il termine nous faccia riferimento alla facoltà umana in entrambe le parti del frammento, per almeno due ragioni: il termine nous, senz'altra precisazione, non può ricoprire un'estensione differente nel primo e nel secondo membro della testimonianza. Il nous è facoltà di intelligere, perciò, per definizione, non essenzialmente in atto. Ma la natura del dio non può essere una facoltà, poiché la natura del dio si risolve completamente nella sua attività, in quella attività che è descritta in Metafisica Lambda 9. Perciò un dio è epekeina ti tou nou, perché non è nous; ma è analogo al nous, perché la sua attività è analoga all'attività più divina nell'uomo, l'attività del nous. Ora, il nous dell'uomo può rimanere allo stato potenziale, al contrario, un dio è atto.

Con ciò, tuttavia, le difficoltà sono state superate. Infatti nessuna formula di questo genere è reperibile nel Corpus. Perciò il criterio abituale, fondato sulla conformità dei termini del frammento aporetico con le altre opere dell'autore, non risulta efficace in questo caso. Siamo di fronte ad un frammento estremamente breve, contenente un pensiero attribuito allo Stagirita, assente dal resto delle opere che ci sono pervenute e in una formulazione che risuona di neoplatonismo. È dunque il caso di concludere che Simplicio, unico testimone del testo, avrebbe a torto attribuito al dialogo aristotelico una dottrina cara alla propria corrente di pensiero? Abbiamo già mostrato come questa accusa sia difficilmente sostenibile, poiché troppe ragioni militano a favore dell'autenticità del testo in questione.

Siamo ben consapevoli che Simplicio appartiene a quei neoplatonici che, pur essendo fedeli a Platone, pensano di poter avvalersi di alcuni aspetti dottrinali di Aristotele. A partire dal III secolo il tempo delle critiche ad 
Aristotele si è ormai concluso, ed è venuto il momento di interpretare $\mathrm{i}$ filosofi classici in un contesto armonizzante. Indubbiamente questa tentazione è viva in Simplicio. Egli non pare dare dimostrazione di possedere un esercitato senso critico nel momento in cui attribuisce ad Aristotele la convinzione che qualche cosa esista al di là dell'essenza (huper ten ousian). L'affermazione è sorprendente se si pensa alla natura dell'ousia nell'opera aristotelica. Essa viene considerata come ciò che veramente è, come l'unità ontologica capace di sussistere, "connotant la propriété fondamentale de ce qu'il est" 60 . Dobbiamo confessare che fatichiamo a vedere un "al di là dell'essenza" nella filosofia di Aristotele. Ma il passo del dialogo Sulla Preghiera non fa riferimento alla sostanza, e soprattutto, in questo caso, il commentatore non sembra aver fatto alcun tentativo di armonizzazione. Normalmente la modifica di un testo da parte di un commentatore, o di un copista, si effettua nella direzione di una semplificazione, della soppressione della difficoltà. Niente del genere in questo testo, al contrario.

In un contesto neoplatonico Simplicio ci offre un frammento aristotelico, nel quale la prima parte si armonizza con il pensiero dello Stagirita, la seconda parte sembra ad esso estranea, e la stessa considerazione si può fare, invertita, in rapporto al neoplatonismo, ossia la prima parte trova difficile collocazione nella filosofia di Plotino, la seconda potrebbe essere una citazione letterale dalle Enneadi di Plotino. Inoltre il frammento Sulla Preghiera ha tutta l'aria di essere un dictum esplicitamente attribuito ad Aristotele.

\section{Conclusione}

L'impressione che abbiamo avuto studiando il frammento è che Aristotele sia alla ricerca della forma nella quale un dio si manifesta nel modo più pieno, privo di potenzialitá o di limitazioni temporali. Tuttavia, dal momento che il divino si dice in molti modi, si rivela necessario compiere una sorta di restrizione che non comporti l'esclusione di altre divinità o di altri enti divini. La seconda parte del frammento vorrebbe dunque apportare una precisazione a quanto detto nell prima parte.

Tuttavia siamo consapevoli che anche la nostra soluzione non è esente da obiezioni. Si potrebbe semplicemente obbiettare: se la seconda parte della

60 Gilson, 1948, p. 47. 
proposizione apporta una precisazione, perché esprimere questa precisazione attraverso una disgiuntiva (e...e)?

La sola risposta che ci pare plausibile, e che non soddisferà la curiosità di chi pone la domanda, è che la formula disgiuntiva sia una formula di modestia, un modo per dichiarare la debolezza umana nel momento in cui voglia esprimere l'ineffabilità divina.

Dopo aver dichiarato che dio è intelligenza in un dialogo consacrato, non dimentichiamolo, alla preghiera, Aristotele avrebbe ben potuto sostenere che un dio si eleva al di sopra della nozione di nous e al di là di ogni parola umana. Abbiamo l'impressione che su questo punto Aristotele sis sia rivelato più attento rispetto ai suoi eredi, quasi avesse guadagnato la consapevolezza che una soluzione dirimente sulla natura di un dio avrebbe annientato la superiorità della divinità. Se è un uomo a parlare di un dio, la sua asserzione non può che essere un discorso propriamente umano, incapace di attingere e circoscrivere la superiorità di un dio. Una dichiarazione sulla natura divina ridurrebbe la divinità ad un artefatto umano e ne modellerebbe le sembianze alla forma mentis del suo artefice.

Questa idea, del resto, non fu estranea neppure ad Aristotele. Nel libro Lambda il filosofo esprime chiaramente il concetto che solo il divino conosce il divino. Come parafrasa bene P. Aubenque: "Aristote se demande ... si Dieu n'est pas le seul théologien et considère comme un défi la prétention de l'homme a partager ... la science du divin" 61 . Che dire dunque di questo ente divino, di cui non possiamo che avere una pallida idea, alla luce di ciò che ci illumina in pochi istanti fuggitivi (mikron chronon) ${ }^{62}$ ?

${ }^{61}$ Aubenque, 1977, p. 364.

${ }^{62}$ Aristotele, Metaph. XII 7, 1072b14; cfr. anche XII 9, $1075 a 8$. 


\section{Referências bibliográficas}

ARISTOTELE. L'éthique á Nicomaque, trad. comm. R.A. Gauthier et J. Y. Jolif. Louvain la Neuve, 1970.

ALLAN, D. J.The Philosophy of Aristotle. London [1952, 1957] 1970.

ARNOU, A. Praxis et theorie: étude de detail sur le vocabulaire et la pensée des Enneades de Plotin. Paris: 1921.

AUBENQUE, P. Le problème de l'être chez Aristote. Paris: [1962, 1966] 1977.

BABUT, D. La religion des philosophes grecs de Thalès aux Stoiciens. Paris: 1974.

BERNAYS, J. Die Dialoge des Aristoteles in ihren Verhaltnis zu seinen übrigen Werken. Berlin: 1863, p. 84-94.

BERTI, E. La filosofia del primo Aristotele. Milano: 1997.

BIGNONE, E. L'Aristotele perduto e la formazione filosofica di Epicuro. Firenze: 1936.

BOTTER, B. Dio e divino in Aristotele. St. Augustin: 2005.

BRÖCKER, W. Aristoteles. Frankfurt am Main: 1964.

BRUNSCHWIG, J. EE I 8, 1218a15-32 et le Peri Thagathou. In: MORAUX, P.; HOLFMGER, D. (Hersg), Untersuchungen zur Eudemischen Ethik. Berlin: 1971.

CHERNISS, H. The riddle of the early academy. New York: [1955] 1962.

CHROUST, A.-H. Some Observations on Aristotle's Doctrine of the Uncreatedness and Indestructibility of the Universe. Rivista critica di storia della filosofia, 32,1977, p. 195-202.

DE FILIPPO, M. Aristotle's Identification of the Prime Mover as a God. The Classical Quarterly, 44, 1994, p. 393-409.

DE VÖGEL, C. J. What was God for Plato? In: Philosophia I, Studies in Greek Philosophy. Assen: 1970.

DES PLACES, E. La religion grecque. Dieux, cultes, rites et sentiment religieux dans la Grece antique. Paris: 1969.

DÜRING, I. Aristotle in the Ancient Biographical Tradition. Göteborg: 1957.

ELDERS, L. Aristotle's Cosmology: A Commentary on the "De Cado". Assen: 1966.

FESTUGIERE, A. J. La révélation d'Hermes Trismégiste, II, Le Dieu cosmique. Paris: 1949.

FRIEDLÄNDER, P. Platon, Eidos, Paideia, Dialogos. Berlin und Leipzig: 1928. GILSON, E. L'être et l'essence. Paris: 1948.

JAEGER, W. Aristoteles. Grundlegung einer Geschichte seiner Entwicklung. Berlin: 1923, trad. it. di G. Calogero, Firenze: [1935] 1955. . The Theology of the Early Greek Phifosophers. Oxford: 1947. 
Aristotle. Foundamentals of the History of His Development. Oxford: 1948.

KOSMAN, A. Aristotle's Metaphysics, Lambda 9, Symposium aristotelicum edited by M. Frede and D. Charles. Oxford: 2000, p. 307- 326.

Aristotle's Prime Mover, in M.L. Gill and J.G. Lennox (eds.), Self Motion from Aristotle to Newton. Princeton: 1994, pp. 135-153.

LEFEVRE, CH. Quinta Natura et la psychologie aristotelicienne. Revue philosophique de Louvain, 69, 1971, p. 543.

MORAUX, P. Les listes anciennes des onvrages d'Aristote. Louvain: 1951.

NORDEN, E. Agnostos Theos. Leipzig: 1913.

NUYENS, F. L'évolution de la psychologie d'Aristote. Louvain: 1948.

ORGAN, T. Randall's Interpretation of Aristotle's Unmoved Mover . The Philosophical Quarterly, 12, 1962, p. 297-305.

PEPIN, J. De la prière. In: Aristote fragments et temoignage., Paris: 1968.

ROBIN, L. La pensée grecque et les origines de l'esprit scientifique. Paris: 1963.

WIELAND, W. Die aristotelische Physik. Untersuchungen über die Grundlegung der Naturwissenschaft. Göttingen: 1962.

E-mail:barbarabotter@gmail.com

Recebido: novembro/2009

Aprovado: dezembro/2009 